International Journal of Pure and Applied Mathematics

Volume 109 No. 1 2016, 119-128

ISSN: 1311-8080 (printed version); ISSN: 1314-3395 (on-line version)

url: http://www.ijpam.eu

doi: 10.12732/ijpam.v109i1.9

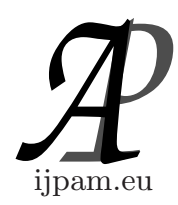

\title{
APPROXIMATION OF THE CUT FUNCTION BY STANNARD AND RICHARD SIGMOID FUNCTIONS
}

\author{
Anton Iliev ${ }^{1}$, Nikolay Kyurkchiev², Svetoslav Markov ${ }^{3}$ \\ ${ }^{1}$ Faculty of Mathematics and Informatics \\ Paisii Hilendarski University of Plovdiv \\ 24 Tsar Assen Str., 4000 Plovdiv, BULGARIA \\ e-mail: aii@uni-plovdiv.bg \\ 1,2,3 Institute of Mathematics and Informatics \\ Bulgarian Academy of Sciences
}

Acad. G. Bonchev Str., Bl. 8, 1113 Sofia, BULGARIA

\begin{abstract}
In this paper we study the uniform approximation of the cut function by smooth sigmoid functions such as Stannard and Richard growth functions. Numerical examples are presented using CAS MATHEMATICA.
\end{abstract}

AMS Subject Classification: $41 \mathrm{~A} 46$

Key Words: sigmoid functions, cut function, step function, stannard growth function, Richard growth function, uniform approximation

This paper is dedicated to the memory of Professor Drumi Baynov.

\section{Introduction}

Several sigmoidal functions (Stannard [10], [16], [19], [23], Richards [13], [17], [20], [22], Chapman-Richards [5]) find numerous applications in various fields related to life sciences, chemistry, physics, artificial intelligence, population dynamics, plant biology, fuzzy set theory, etc.

A practically important class of sigmoid functions is the class of cut functions [6], [7], [9] including the Heaviside step function as a limiting case.

Received: August 1, 2016

Published: September 1, 2016 (c) 2016 Academic Publications, Ltd.

url: www.acadpubl.eu 
Approximation of the cut function by a squashing functions is discussed from various computational and modelling aspects in [15].

We study the uniform approximation of the cut function by Stannard and Richard growth functions. We find an expression for the error of the best uniform approximation.

Present approximation task is extremely actual in connection with detailed precision of the lag phase, growth phase and plateau phase in the growth process [3], [18].

\section{Preliminaries}

\subsection{Sigmoid Functions}

In this work we consider sigmoid functions of a single variable defined on the real line, that is functions of the form $\mathbb{R} \longrightarrow \mathbb{R}$. Sigmoid functions can be defined as bounded monotone non-decreasing functions on $\mathbb{R}$. One usually makes use of normalized sigmoid functions defined as monotone non-decreasing functions $s(t), t \in \mathbb{R}$, such that $\lim s(t)_{t \rightarrow-\infty}=0$ and $\lim s(t)_{t \rightarrow \infty}=1$ (in some applications the left asymptote is assumed to be -1 : $\lim s(t)_{t \rightarrow-\infty}=-1$ ).

\subsection{The Cut and the Stannard Functions}

The cut (ramp) function is the simplest piece-wise linear sigmoid function. Let $\Delta=[\gamma-\delta, \gamma+\delta]$ be an interval on the real line $\mathbb{R}$ with centre $\gamma \in \mathbb{R}$ and radius $\delta \in \mathbb{R}$. A cut function is defined as follows:

Definition. The cut function $c_{\gamma, \delta}$ is defined for $t \in \mathbb{R}$ by

$$
c_{\gamma, \delta}(t)=\left\{\begin{array}{cll}
0, & \text { if } \quad t<\gamma-\delta \\
\frac{t-\gamma+\delta}{2 \delta}, & \text { if } \quad|t-\gamma|<\delta \\
1, & \text { if } \quad t>\gamma+\delta
\end{array}\right.
$$

Note that the slope of function $c_{\gamma, \delta}(t)$ on the interval $\Delta$ is $1 /(2 \delta)$ (the slope is constant in the whole interval $\Delta$ ).

Two special cases and a limiting case are of interest for our discussion in the sequel.

Special case 1. For $\gamma=0$ we obtain the special cut function on the interval $\Delta=[-\delta, \delta]$ : 


$$
c_{0, \delta}(t)=\left\{\begin{array}{cl}
0, & \text { if } \quad t<-\delta, \\
\frac{t+\delta}{2 \delta}, & \text { if } \quad-\delta \leq t \leq \delta \\
1, & \text { if } \quad \delta<t .
\end{array}\right.
$$

Special case 2. For $\gamma=\delta$ we obtain the special cut function on the interval $\Delta=[0,2 \delta]:$

$$
c_{\delta, \delta}(t)=\left\{\begin{array}{cl}
0, & \text { if } \quad t<0 \\
\frac{t}{2 \delta}, & \text { if } \quad 0 \leq t \leq 2 \delta \\
1, & \text { if } \quad 2 \delta<t
\end{array}\right.
$$

A limiting case. If $\delta \rightarrow 0$, then $c_{\delta, \delta}$ tends (in Hausdorff sense) to the Heaviside step function

$$
c_{0}=c_{0,0}(t)=\left\{\begin{array}{cc}
0, & \text { if } t<0 \\
{[0,1],} & \text { if } t=0 \\
1, & \text { if } t>0
\end{array}\right.
$$

which is an interval-valued function [1], [2], [11], [21].

Definition. Define the shifted Stannard function $S_{\gamma}(t)$ with jump at point $\gamma$ as:

$$
S_{\gamma}(t)=\frac{1}{\left(1+e^{\frac{-(\beta+k(t-\gamma))}{m}}\right)^{m}} .
$$

Special case.

$$
\begin{aligned}
S_{\gamma}(t) & =\frac{1}{\left(1+e^{\frac{-(\beta+k(t-\gamma))}{m}}\right)^{m}} \\
S_{\gamma}(\gamma) & =\frac{1}{2}
\end{aligned}
$$

i.e.

$$
\left(1+e^{-\frac{\beta}{m}}\right)^{m}=2
$$




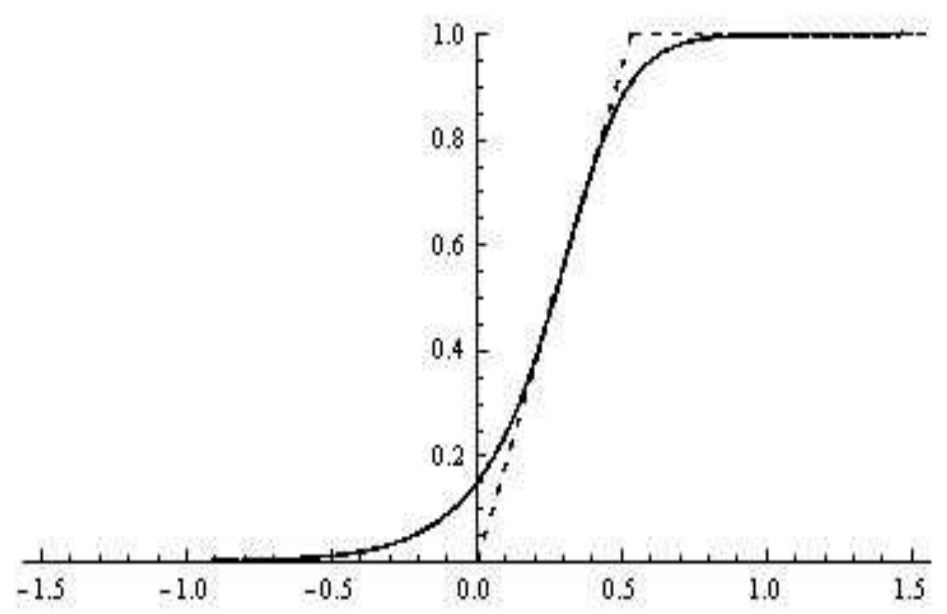

Figure 1: The cut and Stannard function (6)-(7) with $k=5, m=0.5$, $\beta=-0.549306, l=1.875, \gamma=\frac{1}{2 l}=0.266667$, uniform distance $\rho=$ 0.150455 .

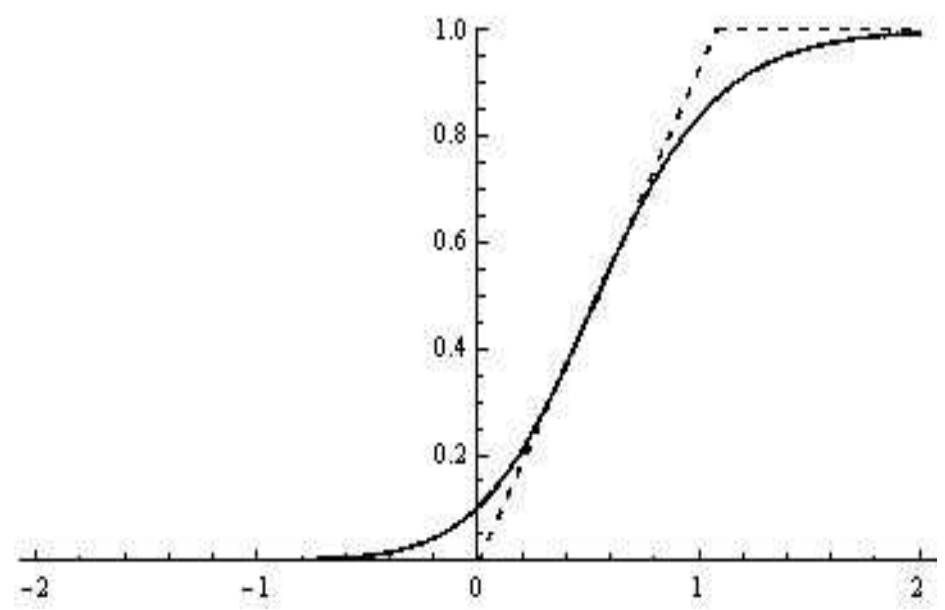

Figure 2: The cut and Stannard function (6)-(7) with $k=5, m=1.5$, $\beta=0.798071, l=0.925099, \gamma=\frac{1}{2 l}=0.540483$, uniform distance $\rho=0.10272$. 


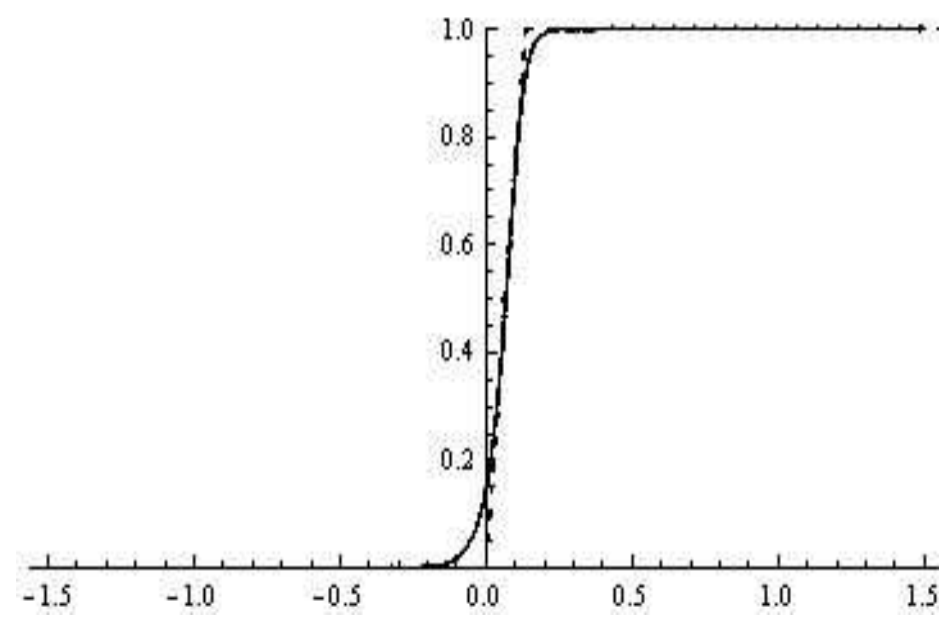

Figure 3: The cut and Stannard function (6)-(7) with $k=20, m=0.5$, $\beta=-0.549306, l=7.5, \gamma=\frac{1}{2 l}=0.0666667$, uniform distance $\rho=$ 0.150455 .

\section{Approximation of the Cut Function by Stannard Function}

We next focus on the approximation of the cut function (1) by shifted Stannard growth function $S_{\gamma}(t)$ defined by $((6)-(7))$.

We note that the slope of $S_{\gamma}(t)$ at $t=\gamma$ is

$$
S_{\gamma}^{\prime}(\gamma)=\frac{k e^{-\frac{\beta}{m}}}{\left(1+e^{-\frac{\beta}{m}}\right)^{m+1}}=l
$$

and the slope of $c_{\delta, \delta}(t)$ at $t=\gamma$ is

$$
c_{\delta, \delta}^{\prime}(\gamma)=\frac{1}{2 \delta}
$$

Let us choose $l=\frac{1}{2 \delta}$. The special Stannard function defined by (6)-(7) has an inflection at point $\left(\gamma, \frac{1}{2}\right)$ (see Fig.1 and Fig.3).

Consider functions (1) and (6) with same centers $\gamma=\delta$, that is functions $c_{\delta, \delta}$ and $S_{\delta}$.

In addition chose $c$ and $S$ to have same slopes at their coinciding centres. 
Then, noticing that the largest uniform distance $\rho$ between the cut and Stannard functions is achieved at the endpoints of the underlying interval $[0,2 \delta]$ we have:

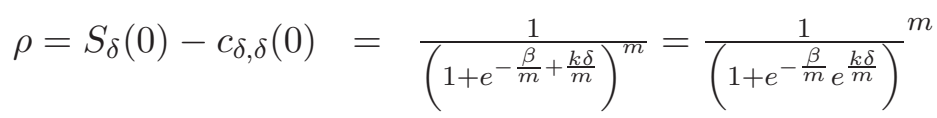

$$
\begin{aligned}
& ={\left.\frac{1}{\left(1+\left(2^{1 / m}-1\right) e^{\frac{2^{1 / m}}{m\left(2^{1 / m}-1\right)}}\right.}\right)}_{m}^{m} \text {. }
\end{aligned}
$$

The above can be summarized in the following

Theorem 1. The function $S_{\gamma}(t)$ defined by (6)-(7): i) is the Stannard function of best uniform one-sided approximation to function $c_{\gamma, \delta}$ in the interval $[\gamma, \infty)$ (as well as in the interval $(-\infty, \gamma])$; ii) approximates the cut function $c_{\gamma, \delta}(t)$ in uniform metric with an error

$$
\rho={\frac{1}{\left(1+\left(2^{1 / m}-1\right) e^{\frac{2^{1 / m}}{m\left(2^{1 / m}-1\right)}}\right)}}^{m} .
$$

Remark. We note that the uniform distance $\rho=\rho(m)$ is an absolute constant that does depends only on the growth parameter $m$ (see Fig. 1 and Fig. 3).

Some computational examples using relation (8) are presented in Table 1.

\begin{tabular}{|c|c|}
\hline$m$ & Uniform distance $-\rho$ \\
\hline 0.5 & 0.150455 \\
1.5 & 0.10272 \\
2 & 0.0927495 \\
2.5 & 0.0860979 \\
5 & 0.071034 \\
10 & 0.0624992 \\
50 & 0.0551328 \\
\hline
\end{tabular}

Table 1: Bounds for $\rho$ computed by (8) for various rates $m$. 


\section{Approximation of the Cut Function by Richard Function}

Definition. Define the special shifted Richard growth function $R_{\gamma}(t)$ with jump at point $\gamma$ as:

$$
R_{\gamma}(t)=\frac{1}{2}\left(\frac{1+m}{1+m e^{-k(t-\gamma)}}\right)^{\frac{1}{m}}
$$

Then we have $R_{\gamma}(\gamma)=\frac{1}{2}$.

We next focus on the approximation of the cut function (1) by shifted Richard growth function $R_{\gamma}(t)$ defined by (9).

We note that the slope of $R_{\gamma}(t)$ at $t=\gamma$ is $R_{\gamma}^{\prime}(\gamma)=\frac{k}{2(1+m)}=l_{1}$ and the slope of $c_{\delta, \delta}(t)$ at $t=\gamma$ is $c_{\delta, \delta}^{\prime}(\gamma)=\frac{1}{2 \delta}$.

Let us choose $l_{1}=\frac{1}{2 \delta}$. The Richard function defined by (9) has an inflection at point $\left(\gamma, \frac{1}{2}\right)$ (see Fig.4 and Fig.5).

Consider functions (1) and (9) with same centers $\gamma=\delta$, that is functions $c_{\delta, \delta}$ and $R_{\delta}$.

In addition chose $c$ and $R$ to have same slopes at their coinciding centres.

Then, noticing that the largest uniform distance $\rho_{1}$ between the cut and Richard functions is achieved at the endpoints of the underlying interval $[0,2 \delta]$ we have:

$$
\begin{aligned}
\rho_{1}=R_{\delta}(0)-c_{\delta, \delta}(0) & =\frac{1}{2}\left(\frac{1+m}{1+m e^{k \gamma}}\right)^{\frac{1}{m}}=\frac{1}{2}\left(\frac{1+m}{1+m e^{\frac{k(1+m)}{k}}}\right)^{\frac{1}{m}} \\
& =\frac{1}{2}\left(\frac{1+m}{1+m e^{1+m}}\right)^{\frac{1}{m}} .
\end{aligned}
$$

The above can be summarized in the following

Theorem 2. The function $R_{\gamma}(t)$ defined by (9): i) is the Richard function of best uniform one-sided approximation to function $c_{\gamma, \delta}$ in the interval $[\gamma, \infty)$ (as well as in the interval $(-\infty, \gamma])$; ii) approximates the cut function $c_{\gamma, \delta}(t)$ in uniform metric with an error

$$
\rho_{1}=\frac{1}{2}\left(\frac{1+m}{1+m e^{1+m}}\right)^{\frac{1}{m}}
$$




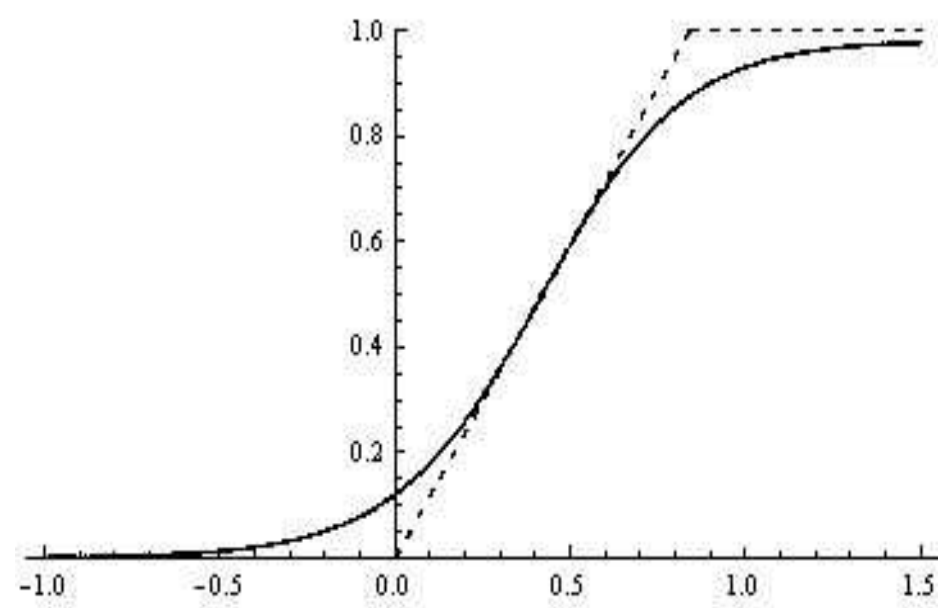

Figure 4: The cut and Richard function (9) with $k=5, m=1.1$, $\gamma=0.42$, uniform distance $\rho_{1}=0.121195$.

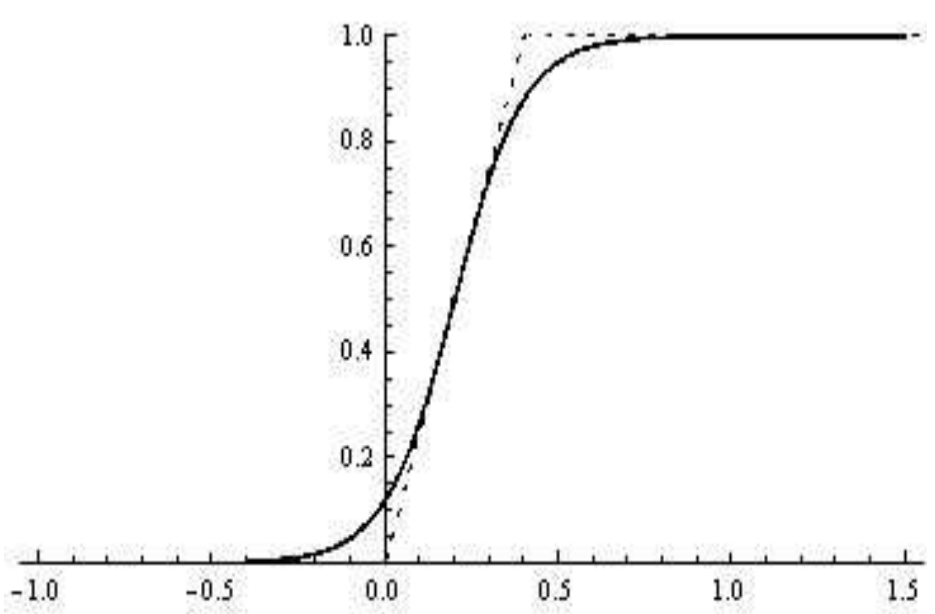

Figure 5: The cut and Richard function (9) with $k=10, m=1.011$, $\gamma=0.201$, uniform distance $\rho_{1}=0.119408$. 


\begin{tabular}{|c|c|}
\hline$m$ & Uniform distance $-\rho_{1}$ \\
\hline 1.1 & 0.121195 \\
1.01 & 0.119408 \\
1.2 & 0.123074 \\
0.5 & 0.107112 \\
0.1 & 0.0937717 \\
0.01 & 0.0991122 \\
\hline
\end{tabular}

Table 2: Bounds for $\rho_{1}$ computed by (10) for various rates $m$.

Some computational examples using relation (10) are presented in Table 2.

For some approximation, computational and modelling aspects, see [12], $[14]$.

Remarks. Usually, the numerical solution of differential equations that describe them, for example, Schnute, Stannard and Richards growth curves is associated with sensitive unstable solutions.

In this sense, could be useful results related to the theory and practice of differential equations [4], [8].

Such views could prove very useful in detail refinement of Lag phase and Plateau phase in elongation processes.

\section{References}

[1] R. Anguelov, S. Markov, Hausdorff Continuous Interval Functions and Approximations, In: M. Nehmeier et al. (Eds), Scientific Computing, Computer Arithmetic, and Validated Numerics, 16th International Simposium, SCAN 2014, Springer, LNCS 9553 (2016), 3-13, doi:10.1007/978-3-319-31769-4

[2] R. Anguelov, S. Markov, B. Sendov, The Set of Hausdorff Continuous Functions - the Largest Linear Space of Interval Functions. Reliable Computing, 12 (2006), 337-363.

[3] P. Arosio, T. P. J. Knowles, S. Linse, On the lag phase in amyloid fibril formation, Physical Chemistry Chemical Physics, 17 (2015), 7606-7618, doi:10.1039/C4CP05563B

[4] D. Bainov, S. Hristova, Differential equations with maxima, CRC Press, New York (2011).

[5] L. Bertalanffy, Quantitative laws in metabolism and growth, J. Quart. Rev. Biol., 32 (1957), 217-231.

[6] J. Dombi, Z. Gera, The Approximation of Piecewise Linear Membership Functions and Lukasiewicz Operators, Fuzzy Sets and Systems, 154, No. 2 (2005), 275-286.

[7] A. Grauel, L. Ludwig, Construction of differentiable membership functions, Fuzzy Sets and Systems, 101 (1999), 219-225. 
[8] S. Hristova, Qualitative investigations and approximate methods for impulsive equations, Nova Science Publishers, New York (2009).

[9] A. Iliev, N. Kyurkchiev, S. Markov, On the approximation of the cut and step functions by logistic and Gompertz functions, Biomath, 4, No. 2 (2015), 2-13.

[10] A. Khamanis, Z. Ismail, Kh. Haron, A. Muhammad, Nonlinear growth models for modeling oil palm yield growth, Journal of Mathematics and Statistics, 1, No. 3 (2005), $225-233$.

[11] W. Kramer, J. Wolff v. Gudenberg, Scientific Computing, Validated Numerics, Interval Methods, Proceedings of the conference Scan-2000/Interval-2000, Kluwer/Plenum (2001).

[12] N. Kyurkchiev, On the Approximation of the step function by some cumulative distribution functions, Compt. rend. Acad. bulg. Sci., 68, No. 12 (2015), 1475-1482.

[13] N. Kyurkchiev, A note on the new geometric representation for the parameters in the fibril elongation process, Compt. rend. Acad. bulg. Sci., 69, No. 8 (2016), 961-970.

[14] V. Kyurkchiev, N. Kyurkchiev, On the Approximation of the Step function by RaisedCosine and Laplace Cumulative Distribution Functions, European International Journal of Science and Technology, 4, No. 9 (2015), 75-84.

[15] N. Kyurkchiev, S. Markov, Sigmoid functions: Some Approximation and Modelling Aspects, LAP LAMBERT Academic Publishing, Saarbrucken (2015), ISBN 978-3-65976045-7.

[16] M. Panik, Growth curve modeling: theory and applications, Wiley, ISBN 978-1-11876404-6, (2014).

[17] F. J. Richards, A Flexible Growth Function for Empirical Use, J. Exp. Bot., 10 (1959), 290-301.

[18] S. Shoffner, S. Schnell, Estimation of the lag time in a subsequent monomer addition model for fibrill elongation, bioRxiv The preprint server for biology (2015), 1-8, doi:10.1101/034900

[19] C. Stannard, A. Williams, P. Gibbs, Temperature/growth relationship for psychotropic food-spoilage bacteria, Food Microbiol., 2 (1985), 115-122.

[20] E. Tjorve, K. Tjorve, A unified approach to the Richards-model family for use in growth analyses: Whu we need only two model forms, J. of Theoretical Biology, 267 (2010), 417-425.

[21] J. H. Van der Walt, The Linear Space of Hausdorff Continuous Interval Functions, Biomath, 2 (2013).

[22] X. Yin, J. Gougrian, E. Lantinga, J. Vos, H. Spiertz, A flexible sigmoid function of determinate growth, Ann. Bot., 91 (2003), 361-371.

[23] M. Zwieterling, I. Jongenburger, F. Rombouts, K. Riet, Modeling of the Bacterial Growth Curve, Appl. and Environmental Microbiology, 56, No. 6 (1990), 1875-1881. 